\title{
Toward detection of terrestrial planets in the habitable zone of our closest neighbor: proxima Centauri ${ }^{\star}, \star \star$
}

\author{
M. Endl ${ }^{1}$ and M. Kürster ${ }^{2}$
}

\author{
${ }^{1}$ McDonald Observatory, University of Texas at Austin, Austin, TX 78712, USA \\ e-mail: mike@astro.as.utexas.edu \\ 2 Max-Planck-Institut für Astronomie, Königstuhl 17, 69117 Heidelberg, Germany
}

Received 26 April 2008 / Accepted 1 July 2008

\begin{abstract}
Context. The precision of radial velocity (RV) measurements to detect indirectly planetary companions of nearby stars has improved to enable the discovery of extrasolar planets in the Neptune and Super-Earth mass range. Detections of extremely low mass planets, even as small as 1 Earth mass or below, in short-period orbits now appears conceivable in ongoing RV planet searches. Discoveries of these Earth-like planets by means of ground-based RV programs will help to determine the parameter $\eta_{\oplus}$, the frequency of potentially habitable planets around other stars.

Aims. In search of low-mass planetary companions we monitored Proxima Centauri (M5V) as part of our M dwarf program. In the absence of a significant detection, we use these data to demonstrate the general capability of the RV method in finding terrestrial planets. For late M dwarfs the classic liquid surface water habitable zone (HZ) is located close to the star, in which circumstances the RV method is most effective. We want to demonstrate that late M dwarfs are ideal targets for the search of terrestrial planets with the RV technique.

Methods. Using the iodine cell technique we obtained differential RV measurements of Proxima Cen over a time span of 7 years with the UVES spectrograph at the ESO VLT. We determine upper limits to the masses of companions in circular orbits by means of numerical simulations.

Results. The RV data of Proxima Cen have a total rms scatter of $3.1 \mathrm{~m} \mathrm{~s}^{-1}$ and a period search does not reveal any significant signals. In contrast to our earlier results for Barnard's star, the RV results for the active M dwarf Proxima Cen are only weakly correlated with $\mathrm{H}_{\alpha}$ line index measurements. As a result of our companion limit calculations, we find that we successfully recover all test signals with $\mathrm{RV}$ amplitudes corresponding to planets with $m \sin i \geq 2-3 M_{\oplus}$ residing inside the $\mathrm{HZ}$ of Proxima Cen with a statistical significance of $>99 \%$. Over the same period range, we can recover $50 \%$ of the test planets with masses of $m \sin i \geq 1.5-2.5 M_{\oplus}$. Based on our simulations, we exclude the presence of any planet in a circular orbit with $m \sin i \geq 1 M_{\text {Neptune }}$ at separations of $a \leq 1$ AU.
\end{abstract}

Key words. stars: individual: proxima Centauri - stars: planetary systems - techniques: radial velocities

\section{Introduction}

Over the next decades we will be able to derive a first estimate of the frequency of stars with a potentially habitable Earth-like planet. This frequency is usually denoted by the parameter $\eta_{\oplus}$. CoRoT and Kepler are two space mission that have the capability to detect "Super-Earths" and even Earth analogs in short-period orbits (CoRoT) and at 1 AU (Kepler) using the transit method. The astrometry mission SIM Planetquest will achieve the sensitivity to detect Earth-like planets around a sample of nearby stars. Also, ground-based Doppler measurements have attained precision levels that make discoveries of planets with a few Earth masses possible (e.g. Lovis et al. 2006). Already the very first extrasolar planets found around the pulsar PSR $1257+12$ (Wolszczan \& Frail 1992), have such small masses, that they qualify as terrestrial planets. However, their formation is likely to have followed a different path than what is currently envisioned for the formation of terrestrial planets around main sequence stars. Besides its obvious astrobiological implications,

\footnotetext{
* Based on observations collected at the European Southern Observatory, Paranal, Chile, programmes 65.L-0428, 66.C-0446, 267.C-5700, 68.C-0415, 69.C-0722, 70.C-0044, 71.C-0498, 072.C0495, 173.C-0606 and 078.C-0829.

$\star \star$ Table 1 is only available in electronic form at

http://www . aanda.org
}

and its crucial role for the design and overall costs of any future TPF/Darwin-type mission, the value of $\eta_{\oplus}$ will also impact our understanding of the formation of terrestrial planets in general (similar to the effect the extrasolar giant planets have on giant planet formation models).

M dwarfs comprise the majority of stars in the solar neighborhood (e.g. Reid et al. 2004) and once their intrinsic faintness is overcome, they represent attractive targets for high precision Doppler surveys. Due to their lower masses the reflex motion of a planet of a given mass is higher than for a solar mass star. Thus, it is no surprise that so far the lowest mass extrasolar planets detected by the Doppler method are all orbiting M dwarfs (Rivera et al. 2005; Udry et al. 2007). The micro-lensing event reported by Beaulieu et al. (2006) is also attributed to lensing by a very low-mass planetary companion to, most likely, an M dwarf host.

In this paper we present 7 years of high precision RV data for our closest neighbor in space: the M5V star Proxima Centauri. We demonstrate that with the data in hand we could have already detected planets with minimum masses as small as 1-2 $M_{\oplus}$. Constraints for giant planetary companions to Proxima Cen using HST Fine Guidance Sensor astrometry were reported by Benedict et al. (1999) and the companion limits based on less precise RV data from the ESO Coudé Echelle Spectrometer planet search were presented by Kürster et al. (1999). The 
combination of both studies already excluded all companions with (minimum) masses higher than $0.8 M_{\text {Jupiter }}$ for the period range 1 to 600 days.

\section{Stellar properties of proxima Cen}

Proxima Centauri (GJ 551, $\alpha$ Cen C, HIP 70890) is an M5Ve dwarf and with a distance of $d=1.29 \mathrm{pc}$ the closest star to the Sun. Hipparcos measured a parallax of $772.33 \pm 2.42$ mas (Perryman et al. 1997). It is still under debate whether Proxima Cen is actually gravitationally bound to the $\alpha$ Cen AB binary (e.g. Wertheimer \& Laughlin 2006). The star exhibits all characteristics of a magnetically active star, like coronal X-ray emission (e.g. Hünsch et al. 1998) and flare activity (e.g. Güdel et al. 2004). Benedict et al. (1998) reported on the photometric variability of Proxima Cen using 4 years of HST FGS photometry. They find a possible rotational period for Proxima Cen of $\approx 84$ days and an activity cycle of $\approx 1100$ days. Recently Cincunegui et al. (2007) presented tentative evidence for a possible activity cycle with a shorter period of $\approx 442$ days.

A crucial parameter for the radial velocity method is the stellar primary mass. Using the $V$-band mass-luminosity relationship of Henry et al. (1999) we derive a mass of $0.108 M_{\odot}$ for Proxima Cen, while the $K$-band mass-luminosity relationship of Delfosse et al. (2000) yields a slightly higher mass of $0.12 M_{\odot}$.

\section{Observations and data reduction}

We observed Proxima Cen with the UVES spectrograph at the ESO VLT-UT2 as part of our ongoing Doppler search for low mass planets around a sample of $40 \mathrm{M}$ dwarfs. We use a slit width of 0.3 arcsec and image slicer \#3 to obtain a resolving power of $R \approx 100000$. The 37 orders on the two CCDs cover a wavelength range from 4950 to $7040 \AA$.

During 76 nights between March 2000 to March 2007 we collected a total of 229 individual spectra of Proxima Cen typically grouped into three consecutive spectra per night. An exposure time of $780 \mathrm{~s}$ was selected for all spectra. The data have an average signal-to-noise ratio of $53.1 \pm 7.7$ (per pixel).

To allow a simultaneous wavelength calibration, as well as the reconstruction of the instrumental profile, we employ the standard iodine $\left(\mathrm{I}_{2}\right)$ cell technique for high precision RV measurements (e.g. Butler et al. 1996). The $\mathrm{I}_{2}$ spectrum has useful reference lines from $\approx 5000$ to $6500 \AA$ that are superimposed on the stellar spectrum.

After bias subtraction, flat-fielding, subtraction of inter-order Echelle background, wavelength calibration, and barycentric correction using the JPL ephemeris DE200 (Standish 1990), we determine differential RVs with our Austral code as described in Endl et al. (2000).

\section{4. $\mathrm{RV}$ results}

One result of the data modeling procedure is a formal internal uncertainty of the RV measurement. This internal uncertainty includes errors due to photon noise, CCD readout noise and "algorithmic" noise in the modeling process to remove all instrumental effects (like spatial and temporal variations in the instrumental profile). For the Proxima Cen data we obtained a mean internal error of $2.34 \pm 0.28 \mathrm{~m} \mathrm{~s}^{-1}$.

As discussed in Kürster et al. (2003) (from hereon called Paper I) we then formed nightly means of the RV measurements

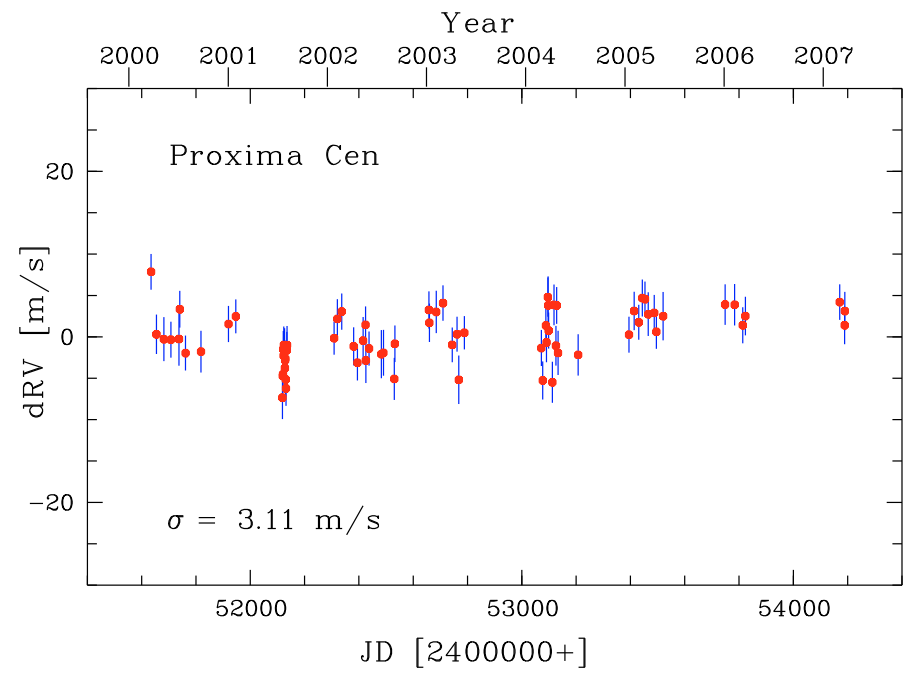

Fig. 1. 7 years of RV measurements for Proxima Cen using $\mathrm{UVES}+\mathrm{I}_{2}$ cell at the ESO VLT. (The secular acceleration has been subtracted.) The data have a total rms of $3.11 \mathrm{~m} \mathrm{~s}^{-1}$ and an average uncertainty of $2.34 \mathrm{~m} \mathrm{~s}^{-1}$.

from the typically three (but occasionally more) consecutive exposures. Removing this "clumpiness" of the data allows for a reliable estimation of the significance of possible signals using the bootstrap randomization method (see Sect. 6). This reduced the total number of data points from 229 to 76 . We then subtracted the expected secular RV acceleration of $0.45 \mathrm{~m} \mathrm{~s}^{-1} \mathrm{yr}^{-1}$, due to the change in perspective to this nearby star, from the data (cf. Paper I). Figure 1 displays our 76 differential RV measurements for Proxima Cen. The velocities are listed in Table 1. The data have a total rms of $3.11 \mathrm{~m} \mathrm{~s}^{-1}$, slightly larger than the measurement uncertainties. The excess RV scatter is thus $2.05 \mathrm{~m} \mathrm{~s}^{-1}$.

We noted that over the past 2 years the RV scatter of Proxima is greatly reduced. While the data taken before 2005 have an rms scatter of $3.04 \mathrm{~m} \mathrm{~s}^{-1}$, the 16 data points we obtained since then have a scatter of only $1.36 \mathrm{~m} \mathrm{~s}^{-1}$. Since neither the instrument nor our data reduction algorithm was changed over this time, this change is likely instrinsic to the star.

\subsection{Period search}

We use the classic Lomb-Scargle periodogram (Lomb 1976; Scargle 1982) to search for possible periodicities in the Proxima Cen RV data. The resulting power spectrum is shown in Fig. 2. The highest peak is located at 363.6 days. There is also an intriguing peak at longer periods from 2000 to 3000 days, but such a long period is comparable to our observing time span. We find no power at the suspected rotation period of $\approx 84 \mathrm{~d}$, and only moderate power at the possible activity cycle period of $\approx 1100 \mathrm{~d}$. To estimate the significance of these signals we use the bootstrap randomization method (e.g. Kürster et al. 1997). After 10000 bootstrap randomizations of our data we find that the $364 \mathrm{~d}$ peak has a formal false-alarm-probability (FAP) of just $0.05 \%$. However, this peak is likely produced by the interplay of the strong 1-year peak in the window function (lower panel in Fig. 2) and the dense data cluster in the middle of 2001. This cluster is systematically lower than the rest of the points. If we reduce the weight of these points by averaging them we find a reduction in power of the 364-d peak. (The 1-yr peak disappears completely if we remove the entire cluster from the time series.) We will discuss the cause of the systematic blue-shift of these 


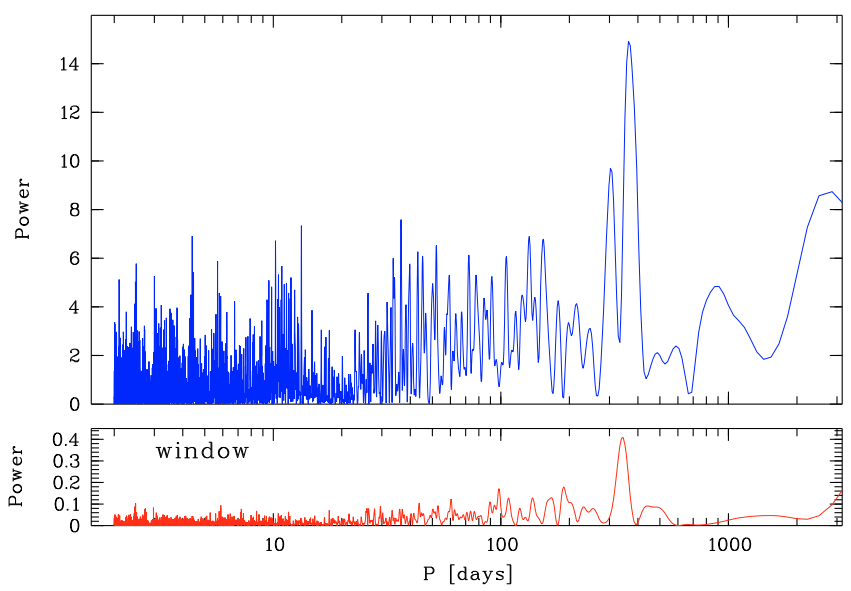

Fig. 2. Lomb-Scargle periodogram of the Proxima Cen RV data. The upper panel shows the power spectrum and the lower panel displays the window function. The 1-year peak in our window function is quite dominant. Using the bootstrap method to estimate the significance of the peak shows that no significant signal, except the peak close to 1 year, is present in our data.

points in the next section. No other peak in the periodogram has a FAP less than $1 \%$.

\section{5. $\mathrm{H}_{\alpha}$ line index measurements}

As Proxima Cen is a known active flare star we suspected that the excess scatter might be caused by stellar activity and that we would find a correlation of the RV data with an activity indicator. In the same fashion as for Barnard's star (see Paper I for details) we determined a line strength index for $\mathrm{H}_{\alpha}$ and, as a comparison, an index for a nearby $\mathrm{CaI}$ line whose strength should not depend on the activity level of the star. While for a relatively inactive M dwarf like Barnard's star the chromospheric emission is seen mostly as a small "filling in" of the $\mathrm{H}_{\alpha}$ line core, in the case of Proxima Cen it is seen at all times as a strong emission feature above the continuum. We calculate the line index as the flux inside a region of $\pm 15 \mathrm{~km} \mathrm{~s}^{-1}$ of the center of the line normalized to the flux of 2 adjacent spectral regions. As a check against systematic errors we do the same for the CaI line at $6572.795 \AA$. We determine the line index for each individual spectrum and then average the values for each night in the same manner as for the $\mathrm{RV}$ results.

Proxima Cen reveals a large amount of variability in $\mathrm{H}_{\alpha}$. The average $\mathrm{H}_{\alpha}$ line index is 2.89 with a standard deviation of 0.90 $(31 \%)$, while the $\mathrm{CaI}$ indices is indeed quite constant. The mean $\mathrm{CaI}$ index is 0.57165 with a standard deviation of $0.0103(1.8 \%)$. The amount of variability of the $\mathrm{H}_{\alpha}$ index is more than 4 times larger than what we have found in Barnard's star (7.6\%).

However, a period search in the line index data did not find a significant signal, likely due to the stochastic nature of the variations (flaring). We find the highest power of 7.13 at a period of 25.7 days with an estimated FAP of $35 \%$.

Figure 3 displays the $\mathrm{H}_{\alpha}$ line indices as a function of the $\mathrm{RV}$ results. We adopt as the error of the line index the rms of the individual measurements that were averaged. We find a weak correlation between the $\mathrm{H}_{\alpha}$ data and the RV results. The linear correlation coefficient is -0.23 resulting in a $4.8 \%$ probability that the null-hypothesis, that these two values are not correlated, is correct. A linear fit to the data yields a slope of $-0.71 \pm 0.30 \mathrm{~m} \mathrm{~s}^{-1} \mathrm{H}_{\alpha}-$ index $^{-1}$. The scatter around this fit is $3.03 \mathrm{~m} \mathrm{~s}^{-1}$ (as compared to the overall scatter of $3.11 \mathrm{~m} \mathrm{~s}^{-1}$ ). At

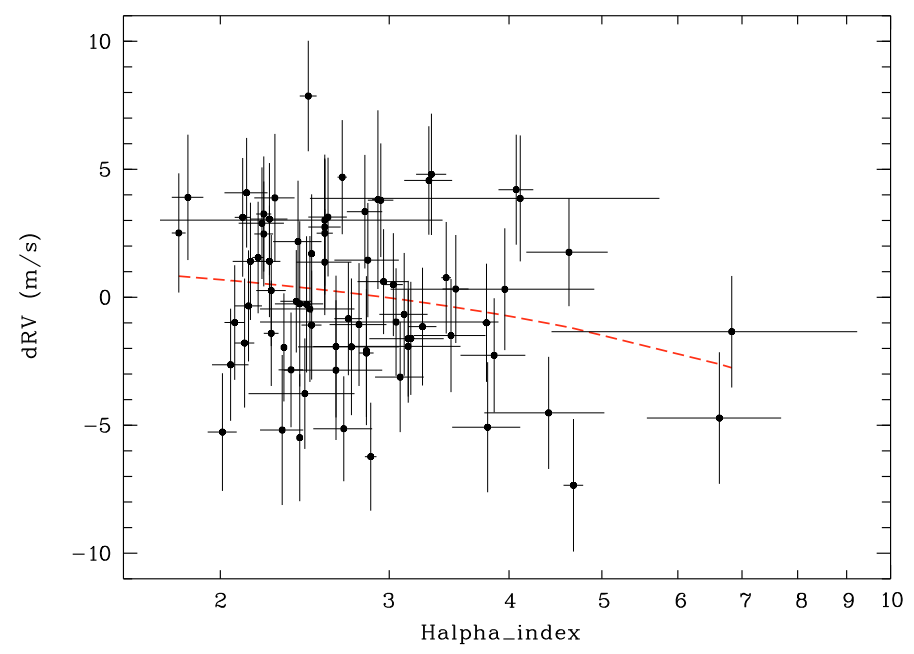

Fig. 3. Proxima Cen $\mathrm{H}_{\alpha}$ line index measurements as a function of RV. The error bar of the line index is the rms of the individual measurements (within one night) binned to form one data point, in the same manner as the RV data. There appears to be a weak correlation between the $\mathrm{H}_{\alpha}$ index and the RV data. The linear correlation coefficient is -0.23 . A fit to the data is shown as a dashed line. The residual RV scatter around this slope is $3.03 \mathrm{~m} \mathrm{~s}^{-1}$.

least in a qualitative way this confirms the trend we have found in Paper I: spectra with a higher $\mathrm{H}_{\alpha}$ index appear blue shifted. However, the correlation for Proxima Cen is much weaker and the slope is much less significant than in the case of Barnard's star. (See the detailed discussion in Paper I why this might indicate a general convective redshift for $\mathrm{M}$ dwarfs.) It is interesting to note that despite the fact that Proxima Cen is a much more active star, its activity level does not produce a higher RV excess scatter ("jitter") than in Barnard's star.

Proxima Cen appears to have been slightly more active during the intensive campaign in summer 2001. The average $\mathrm{H}_{\alpha}$ line index of this data cluster (16 points) is $3.33 \pm 0.29$ (error of the mean) while the mean of the rest of the data is $2.77 \pm 0.11$ (60 points). This small $(1.4 \sigma)$ difference might be the explanation why this group of measurements is systematically blueshifted from the rest.

\section{Limits for planetary companions}

With the absence of a clear RV signal of planets in the Proxima Cen data we pose the question which type of planets we could have already detected and hence we can exclude. We compute mass upper limits with a method of injecting and recovering signals into the original RV data set (Endl et al. 2001). We start with the assumption that the residual scatter in our data is the best representation of the errors and use the bootstrap randomization method to determine the statistical significance of the recovered signal. We intentionally do not correct the RV data with the small activity related slope we determined in the previous section, in order to determine our detection efficiency also in the presence of this source of correlated noise. The mass limit for a given period is set at the lowest signal amplitude where the signal is recovered at all trial phase angles with a significance of $>99 \%$. The test signals were generated at 16 different phase angles, each shifted by $\pi / 8$. We restrict the simulations to circular orbits. Limits derived for eccentric orbits are typically higher (see e.g. Wittenmyer et al. 2006, for a comparison between $e=0$ and $e \leq 0.6$ limits), but in this case we are particularly interested 


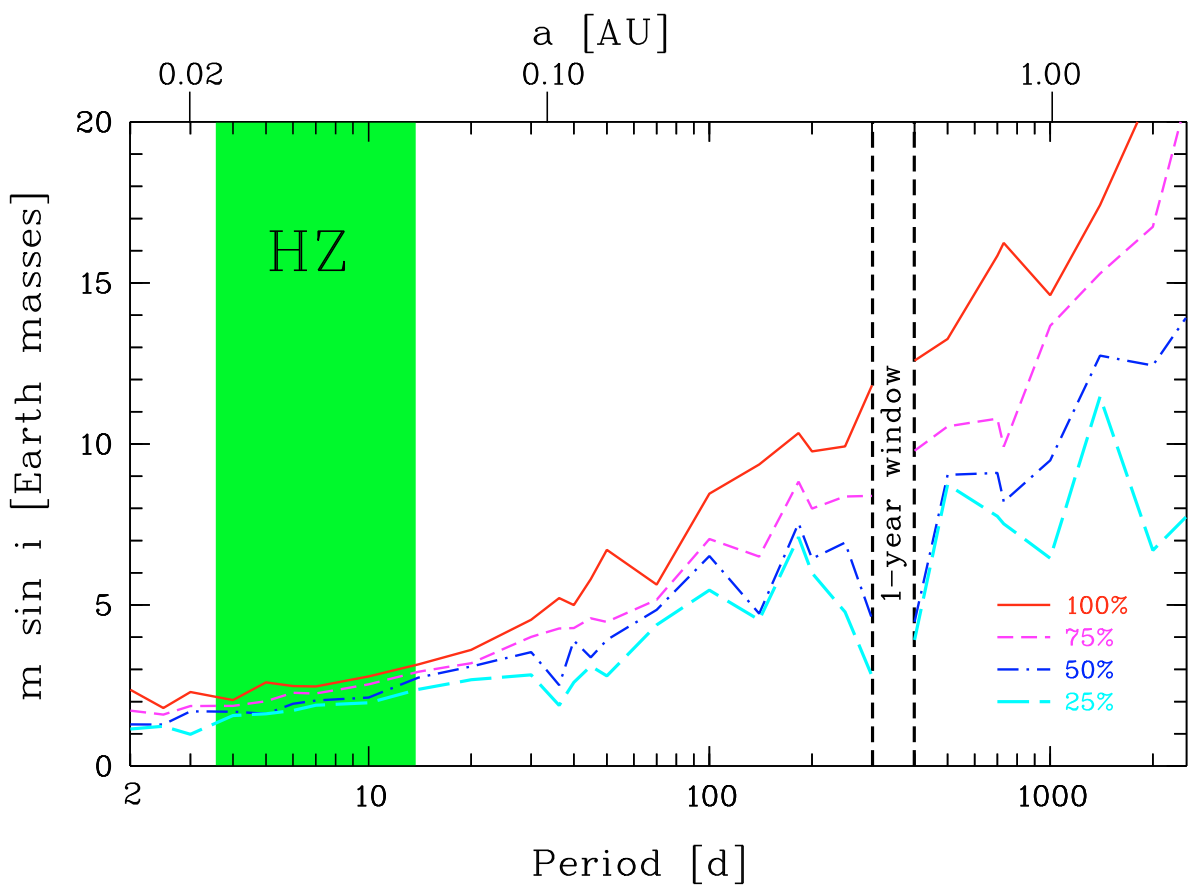

Fig. 4. Mass upper limits for planets in circular orbits around Proxima Cen based on our numerical simulations. All test signals with amplitudes corresponding to masses on and above the solid line were recovered with a $>99 \%$ significance. The solid, dashed, dashed-dotted and long-dashed lines show the mass range where we successfully recovered $100,75,50$ and $25 \%$ of the test signals. The shaded area labelled "HZ" displays the approximate location of the classic liquid water habitable zone (after Kasting et al. 1993). The region labelled "1-year window" shows the period range that we excluded from our simulations. in planets that reside inside the habitable zone (HZ) all the time. An additional constraint is that for a successful recovery of the signal the peak in the power spectrum has to be at (or near) the correct input period. This is especially important for Proxima where the 1-yr period at the maximum of the window function appears frequently as the dominant signal. In a conservative approach we adopt the higher value of $0.12 M_{\odot}$ for the stellar mass (see Sect. 2).

Figure 4 shows the mass upper limits for planets based on this method. The solid line represents the mass range where all test signals were recovered at (or near) the correct period with a significance level of $>99 \%$. The shaded area delimits the classic liquid surface water HZ after Kasting et al. (1993). For such a late type star the HZ is very close to the host star, for Proxima Cen it ranges from 0.022 to about 0.054 AU, corresponding to orbital periods from 3.6 to 13.8 days.

We can not determine reliable upper limits in the period range close to 1 year. The fact that a strong signal at this period is present in the original data leads to the effect that even a zero amplitude input signal in phase with the original signal will always lead to a successful detection. We therefore exclude the region of $300<P>400$ days from our simulations. (This excluded part of the mass-period diagram is shown as "1-year window" in Fig. 4.)

For periods outside the gap at 1 year we can exclude all planets with $m \sin i \geq 16 M_{\oplus}$, i.e. planets with minimum masses greater than Neptune's mass, out to 1 AU. For periods less than 100 days (or $a<0.21 \mathrm{AU}$ ) we could have detected all "SuperEarths" with $m \sin i \geq 8.5 M_{\oplus}$ ). For the HZ of Proxima Cen we can rule out the presence of all planets with $m \sin i \geq 2-3 M_{\oplus}$ in circular orbits. In order not to confuse these upper limits with our actual sensitivity to low mass planets we also show the mass range where 75,50 and $25 \%$ of the test signals were recovered with a statistical significance of $>99 \%$ (dashed, dash-dotted and long-dashed lines in Fig. 4). This demonstrates that we already had a $50 \%$ chance to detect planets with masses as low as $m \sin i=1.5 M_{\oplus}$ inside the HZ of Proxima Cen and slightly inside the inner edge of the $\mathrm{HZ}$ even down to $m \sin i \approx 1.2 M_{\oplus}$. Over the entire range of periods (with the exception of the 1 -yr window) we are $100 \%(75,50$ and $25 \%)$ complete for
RV signals with semi-amplitudes of $K=4.44 \pm 0.33 \mathrm{~m} \mathrm{~s}^{-1}$ $\left(3.47 \pm 0.32,2.56 \pm 0.44\right.$ and $2.01 \pm 0.60 \mathrm{~m} \mathrm{~s}^{-1}$, respectively).

\section{Discussion}

We began our VLT/UVES Doppler survey to search for terrestrial planets in the HZ of M dwarfs already in 2000 . We presented first estimates of the survey sensitivity for low mass planets in Paper I for Barnard's star and in Endl et al. (2003) for Proxima Cen. With the results from this paper we demonstrate that the discovery of $m \sin i \approx 1 M_{\oplus}$ is within our grasp. Since sensitivity is a function of RV precision, number of measurements and sampling, adding more points to the existing data string in a pseudo-random fashion, will allow us to improve the detection sensitivity over time.

Guedes et al. (2008) discuss an RV search for terrestrial planets in the HZ of $\alpha$ Cen B. They conclude that the detection of a $1.7 M_{\oplus}$ planet will require continuous monitoring of the star over at least 3 years and the accumulation of nearly 100000 independent measurements with a precision of $\approx 3 \mathrm{~m} \mathrm{~s}^{-1}$. We have shown here that by switching targets to Proxima Cen the same feat can be achieved with less than 100 data points. The main difference being the lower mass of the star as well as the much shorter orbital periods of $\mathrm{HZ}$ planets.

When interpreting the mass limits, the reader should bear in mind that we only considered circular orbits. Limits for planets on eccentric orbits are typically slightly higher (see e.g. Wittenmyer et al. 2006). Planets with masses above our mass threshold for circular orbits can still exist around Proxima Cen on eccentric orbits. We also considered only the case of a single planet. The RV signals of a multi-planet system with several lowmass bodies, is likely to be more difficult to detect by a pure periodogram analysis (depending on their period spacing and mass ratios) and requires a significantly larger data sets. Simulations to determine the mass limits for multiple planets is beyond the scope of this paper.

The question whether terrestrial planets inside the $\mathrm{HZ}$ of M dwarfs are suitable for life has been discussed extensively in the literature (see e.g. Tarter et al. 2007; Scalo et al. 2007, for a review of this subject). At first glance, $\mathrm{M}$ dwarf $\mathrm{HZ}$ planets 
did not appear to be attractive objects for astrobiology, because their small semi-major axes would lead to tidal locking into synchronous rotation and presumably to atmospheric collapse at the night side of the planet. However, more detailed climate studies have shown that energy transfer between the day and night side of such a planet can prevent atmospheric collapse (Joshi et al. 1997). Furthermore, the long main-sequence life times of M dwarfs would presumably lead to stable environments on such planets on very long time scales. Recently, Lissauer (2007) and Raymond et al. (2007) discuss the potential of habitable M dwarf $\mathrm{HZ}$ planets in the context of their formation and in particular water delivery and find that these planets might be devoid of volatiles and thus less suitable for life.

One of the drawbacks of the RV technique is the sin $i$ ambiguity, we measure only minimum mass values while the true mass remains unknown. But mass is, of course, a crucial parameter for the characterization of a planet. Low mass planets inside the $\mathrm{HZ}$ of $\mathrm{M}$ dwarfs have a higher probability to transit their host star, due to their smaller semimajor axes. Moreover, the transit depth for a given planet will be deeper for $M$ dwarfs, than for earlier spectral types (see e.g. Gillon et al. 2007). Finding more short periodic transiting Super-Earths around M dwarfs will thus potentially increase the sample of planets for which we can distinguish their internal composition by putting them on a massradius diagram (e.g. Selsis et al. 2007; Valenica et al. 2007).

We have shown that finding terrestrial planets in short periods around $\mathrm{M}$ dwarfs is possible even without an $\mathrm{RV}$ precision of $1 \mathrm{~m} \mathrm{~s}^{-1}$ or better. $\mathrm{M}$ dwarfs with masses less than $0.2 M_{\odot}$ are numerous in the solar neighborhood. However, the majority of them are too faint in the V-band, to be accessed by traditional Doppler surveys, working in the optical spectral range. A near infrared high resolution spectrograph (e.g. Guenther et al. 2006) that can obtain an RV precision of $2-3 \mathrm{~m} \mathrm{~s}^{-1}$ would thus be the ideal tool to carry out a search for nearby $m \sin i<1 M_{\oplus}$ planets.

\section{Conclusions}

1. We present 7 years of high precision RV data for our closest neighbor in space, the M5V star Proxima Cen, obtained with UVES $+I_{2}$ cell at the ESO VLT/UT2. We detect no significant periodicities (except close to the 1-yr peak in the window function) that can be attributed to orbiting companions.

2. Using the same set of spectra we measure an $\mathrm{H}_{\alpha}$ line index to estimate the magnetic activity level of Proxima Cen. These line indices show a large amount of scatter due to flaring activity and are only weakly correlated with the RV results.

3. Based on numerical simulations we demonstrate that we could have already detected all planets with $m \sin i=2-$ $3 M_{\oplus}$ on circular orbits inside the classic habitable zone of Proxima Cen (assuming a stellar mass of $0.12 M_{\oplus}$ ).
Acknowledgements. This material is based on work supported by the National Aeronautics and Space Administration under Grants NNG04G141G, NNG05G107G issued through the Terrestrial Planet Finder Foundation Science program and Grant NNX07AL70G issued through the Origins of Solar Systems Program. We are grateful to the ESO OPC and ESO DDTC for generous allocation of observing time to our UVES M dwarf project. A big thank you to the Paranal Science Operations Team who performed all the service mode observations. The comments of the anonymous referee were very helpful to improve the manuscript. We thank Artie P. Hatzes, William D. Cochran, Günther Wuchterl and John Scalo for interesting discussions on M dwarf planets. Sebastian Els provided the code for the barycentric correction for the UVES data and Frédéric Rouesnel and Mathias Zechmeister helped with parts of the spectra extraction.

\section{References}

Beaulieu, J.-P., Bennet, D. P., Fouqué, P., et al. 2006, Nature, 439, 437 Benedict, G. F., McArthur, B., Nelan, E., et al. 1998, AJ, 116, 429 Benedict, G. F., McArthur, B., Chappell, D. W., et al. 1999, AJ, 118, 1086 Butler, R. P., Marcy, G. W., Williams, E., et al. 1996, PASP, 108, 500 Cincunegui, C., Diaz, R. F., \& Mauas, P. J. D. 2007, A\&A, 461, 1107 Delfosse, X., Forveille, T., Segransan, D., et al. 2000, A\&A, 364, 217 Endl, M., Kürster, M., \& Els, S. 2000, A\&A, 362, 585

Endl, M., Kürster, M., Els, S., Hatzes, A. P., \& Cochran, W. D. 2001, A\&A, 374, 675

Endl, M., Kürster, M., Rouesnel, F., et al. 2003, in Scientific Frontiers in Research on Extrasolar Planets, ed. D. Deming \& S. Seager (San Francisco: ASP), ASP Conf. Ser., 294, 75

Gillon, M., Pont, F., Demory, B.-O., et al. 2007, A\&A, 472, 13

Güdel, M., Audard, M., Reale, F., Skinner, S. L., \& Linksy, J. L. 2004, A\&A, 416, 713

Guedes, J. M., Rivera, E. J., Davis, E., et al. 2008, ApJ, 679, 1582

Guenther, E. W., Martin, E. L., Barrado y Navascues, D., \& Laux, U. 2006, Proceedings of Tenth Anniversary of 51 Peg-b: Status of and prospects for hot Jupiter studies, ed. L. Arnold, F. Bouchy \& C. Moutou, I 326

Henry, T. J., Franz, O. G., Wasserman, L. H., et al. 1999, ApJ, 512, 864

Hünsch, M., Schmitt, J. H. M. M., Sterzik, M. F., Voges, W. 1998, A\&AS, 135, 319

Joshi, M. M., Haberle, R. M., \& Reynolds, R. T. 1997, Icarus, 129, 450

Kasting, J. F., Whitmire, D. P., \& Reynolds, R. T. 1993, Icarus, 101, 108

Kürster, M., Schmitt, J. H. M. M., Cutispoto, G., \& Dennerl, K. 1997, A\&A, 320,831

Kürster, M., Hatzes, A. P., Cochran, W. D., et al. 1999, A\&A, 344, L5

Kürster, M., Endl, M., Rouesnel, F., et al. 2003, A\&A, 403, 1077

Lissauer, J. J. 2007, ApJ, 660, 149

Lomb, N. R. 1976, Ap\&SS, 39, 477

Lovis, C., Mayor, M., Pepe, F., et al. 2006, Nature, 441, 305

Perryman, M. A. C., Lindegren, L., Kovalevsky, J., et al. 1997, A\&A, 323, L49

Raymond, S. N., Scalo, J., \& Meadows, V. 2007, ApJ, 669, 606

Reid, I. N., Cruz, K. L., Allen, P., et al. 2004, AJ, 128, 463

Rivera, E. J., Lissauer, J. J., Butler, R. P., et al. 2005, ApJ, 634, 625

Scalo, J., Kaltenegger, L., Segura, A. G., et al. 2007, AsBio, 7, 85

Scargle, J. D. 1982, ApJ, 263, 835

Selsis, F., Chazelas, B., Borde, P., et al. 2007, Icarus, 191, 453

Standish, E. M. 1990, A\&A, 233, 252

Tarter, J. C., Backus, P. R., Mancinelli, R. L., et al. 2007, AsBio, 7, 30

Udry, S., Bonfils, X., Delfosse, X., et al. 2007, A\&A, 469, 43

Valencia, D., Sasselov, D. D., O'Connell, R. J. 2007, ApJ, 665, 1413

Wertheimer, J. G., \& Laughlin, G. 2006, AJ, 132, 1995

Wittenmyer, R. A., Endl, M., Cochran, W. D., et al. 2006, AJ, 132, 177

Wolszczan, A., \& Frail, D. A. 1992, Nature, 355, 145 
M. Endl and M. Kürster: Toward detection of terrestrial planets in the habitable zoneof our closest neighbor: proxima Centauri, Online Material p 1

Table 1. Differential radial velocities of Proxima Cen from VLT/UVES Table 1. continued.

\begin{tabular}{|c|c|c|}
\hline JD [2 $400000+]$ & $\mathrm{dR} V\left[\mathrm{~m} \mathrm{~s}^{-1}\right]$ & $\sigma\left[\mathrm{m} \mathrm{s}^{-1}\right]$ \\
\hline 51634.7343 & 7.86 & 2.16 \\
\hline 51654.6206 & 0.31 & 2.38 \\
\hline 51681.7798 & -0.28 & 2.66 \\
\hline 51707.6289 & -0.34 & 2.17 \\
\hline 51737.5345 & -0.26 & 3.23 \\
\hline 51740.5824 & 3.34 & 2.22 \\
\hline 51761.5579 & -1.96 & 2.11 \\
\hline 51818.4977 & -1.79 & 2.52 \\
\hline 51919.8414 & 1.55 & 2.18 \\
\hline 51946.8066 & 2.47 & 2.05 \\
\hline 52118.5200 & -7.35 & 2.59 \\
\hline 52119.5002 & -4.72 & 2.57 \\
\hline 52120.4995 & -4.52 & 2.19 \\
\hline 52121.5046 & -1.50 & 2.21 \\
\hline 52122.5041 & -2.27 & 2.23 \\
\hline 52123.5158 & -0.99 & 2.24 \\
\hline 52124.5091 & -1.62 & 2.26 \\
\hline 52125.5043 & -1.92 & 2.20 \\
\hline 52126.5086 & -1.09 & 2.13 \\
\hline 52127.5023 & -3.77 & 2.16 \\
\hline 52128.5090 & -2.84 & 2.24 \\
\hline 52129.5172 & -2.64 & 2.19 \\
\hline 52130.5194 & -5.14 & 2.05 \\
\hline 52131.5036 & -6.23 & 2.11 \\
\hline 52134.5201 & -1.61 & 2.21 \\
\hline 52135.5366 & -1.00 & 2.31 \\
\hline 52308.8586 & -0.16 & 2.00 \\
\hline 52320.8412 & 2.17 & 2.38 \\
\hline 52336.8520 & 3.05 & 2.19 \\
\hline 52380.6817 & -1.15 & 2.30 \\
\hline 52394.7349 & -3.12 & 2.15 \\
\hline 52415.6011 & -0.46 & 2.85 \\
\hline 52424.6250 & 1.45 & 2.23 \\
\hline 52425.6540 & -2.85 & 2.73 \\
\hline 52437.5534 & -1.41 & 2.06 \\
\hline 52482.6464 & -2.09 & 2.91 \\
\hline 52490.5975 & -1.93 & 2.77 \\
\hline 52530.5056 & -5.08 & 2.54 \\
\hline 52532.5082 & -0.84 & 2.21 \\
\hline 52657.8318 & 3.25 & 2.25 \\
\hline 52659.7972 & 1.70 & 2.32 \\
\hline 52684.8259 & 3.01 & 2.56 \\
\hline 52709.6935 & 4.08 & 2.14 \\
\hline 52743.8400 & -0.97 & 2.10 \\
\hline 52761.5228 & 0.32 & 2.11 \\
\hline 52767.9010 & -5.19 & 2.93 \\
\hline 52788.5002 & 0.49 & 2.01 \\
\hline 53071.8829 & -1.35 & 2.18 \\
\hline 53076.8916 & -5.27 & 2.30 \\
\hline 53088.8399 & 1.37 & 2.07 \\
\hline 53090.8887 & -0.67 & 2.40 \\
\hline 53095.8975 & 4.80 & 2.37 \\
\hline 53096.8803 & 3.81 & 3.49 \\
\hline 53098.7983 & 0.76 & 2.18 \\
\hline 53112.6938 & -5.49 & 2.48 \\
\hline 53118.5595 & 3.86 & 2.46 \\
\hline 53125.6977 & -1.07 & 2.40 \\
\hline 53128.6031 & 3.79 & 2.22 \\
\hline 53133.5396 & -1.94 & 2.67 \\
\hline 53207.4883 & -2.18 & 2.50 \\
\hline
\end{tabular}

\begin{tabular}{ccc}
\hline \hline $\mathrm{JD}[2400000+]$ & $\mathrm{dR} V\left[\mathrm{~m} \mathrm{~s}^{-1}\right]$ & $\sigma\left[\mathrm{m} \mathrm{s}^{-1}\right]$ \\
\hline 53394.8511 & 0.26 & 2.17 \\
53413.8344 & 3.13 & 2.32 \\
53430.8431 & 1.76 & 2.11 \\
53443.8731 & 4.69 & 2.23 \\
53453.7691 & 4.56 & 2.12 \\
53465.8306 & 2.74 & 2.64 \\
53487.6901 & 2.89 & 2.18 \\
53495.7335 & 0.61 & 2.05 \\
53520.5247 & 2.50 & 2.92 \\
53748.8611 & 3.90 & 2.45 \\
53783.7137 & 3.88 & 2.50 \\
53813.6861 & 1.40 & 2.18 \\
53823.6115 & 2.51 & 2.33 \\
54170.8614 & 4.20 & 2.15 \\
54188.8877 & 1.40 & 2.29 \\
54189.7161 & 3.12 & 2.32 \\
\hline
\end{tabular}

